\title{
Conformally flat black holes in Poincaré gauge theory
}

\author{
M. Blagojević and B. Cvetković* \\ Institute of Physics, University of Belgrade \\ Pregrevica 118, 11080 Belgrade, Serbia
}

September 6, 2021

\begin{abstract}
General criteria for the existence of conformally flat Riemannian solutions in 3D Poincaré gauge theory without matter are formulated. Using these criteria, we show that the Oliva-Tempo-Troncoso black hole, a solution of the Bergshoeff-Hohm-Townsend gravity, is also an exact vacuum solution of the Poincaré gauge theory. The related conserved charges, calculated from the Hamiltonian boundary term, are shown to satisfy the first law of black hole thermodynamics. The form of the boundary term is verified by using the covariant Hamiltonian approach.
\end{abstract}

\section{Introduction}

The use of three-dimensional (3D) gravitational models in the Poincaré gauge theory (PGT), the first properly formulated gauge theory of gravity [1, 2, 3, 4, started in the early 1990s, when Mielke and Baekler formulated a topological model of 3D gravity with torsion [5]. Studies of different aspects of the model made a significant contribution to a proper understanding of the influence of torsion on the gravitational dynamics; for a recent review, see Blagojević and Hehl [4, chapter 17. But, as time went on, it eventually became clear that transition to the level of quadratic PGT Lagrangians is needed, as the existence of propagating torsion modes offers a more realistic insight into the dynamical role of torsion; for more details, see Helayël-Neto et al. [6], Blagojević and Cvetković [7].

It is well known that classical solutions are an important tool for exploring dynamical content of gravitational theories, including the quadratic PGT [4]. Looking at what has been done in $3 \mathrm{D}$, one should note that the model can accommodate exact torsion waves [8] and a Vaidya-like solution with torsion [9]. Quite interestingly, the methods used to construct Siklos waves in [8] are recently generalized to 4D [10].

In order to properly understand the complex dynamical structure of PGT, powerful Lagrangian and Hamiltonin formalisms have been developed, see Obukhov [3], Chen et al. [11, and Ref. [2, 4]. This machinery is very useful not only for genuine PGT problems, characterized by a nonvanishing torsion, but also in studying torsion-free solutions of

\footnotetext{
*Email addresses: mb@ipb.ac.rs, cbranislav@ipb.ac.rs
} 
PGT. On the other hand, quite recently [9] we noticed that the issue of conserved charges of the Oliva-Tempo-Troncoso (OTT) black hole [12], a solution of the Bergshoeff-HohmTownsend (BHT) massive gravity [13] for the special choice of parameters, is not completely settled in the literature, see [14, 15, 16. Such a situation motivated us to reconsider the OTT black hole as a Riemannian (torsion-free) solution of PGT, and try to find the conserved charges, energy and angular momentum, relying on the full power of the constrained Hamiltonian formalism. The analysis is based on deriving the Hamiltonian boundary term, the values of which correctly reproduce the conserved charges.

The paper is organized as follows. In section 2, we use the PGT field equations to study dynamical properties of Riemannian solutions. In particular, we show that: (i) for a specific condition on the coupling constants, Riemannian solutions of PGT are conformally flat, and (ii) any conformally flat solution of the BHT gravity is also a solution of PGT. The results are used in section 3 to prove that the static OTT black hole is a solution of PGT. In section 4, we introduce a set of asymptotic conditions naturally associated to this black hole, and use the constrained Hamiltonian formalism to construct the improved canonical generator $\tilde{G}$, acting on the related phase space [17]. The form of the boundary term in $\tilde{G}$ is shown to be directly related to the OTT asymptotic conditions, and the conserved charges, defined as the values of $\tilde{G}$, are proved to be fully compatible with the first law of black hole thermodynamics. In section 5 , the same approach is used to analyze the rotating OTT black hole, and in section 6 , we summarize our results and verify the form of the boundary term by comparing it to the generalized covariant formula proposed by So [18]. Appendices contain some technical details.

Our conventions are the same as in Ref. [9]: the Latin indices $(i, j, k, \ldots)$ refer to the local Lorentz frame, the Greek indices $(\mu, \nu, \rho, \ldots)$ refer to the coordinate frame, $b^{i}$ is the orthonormal triad (coframe 1-form), $\omega^{i j}$ is the Lorentz connection (1-form), the respective field strengths are the torsion $T^{i}=d b^{i}+\omega^{i}{ }_{m} \wedge b^{m}$ and the curvature $R^{i j}=d \omega^{i j}+\omega^{i}{ }_{k} \wedge \omega^{k j}$ (2-forms), the frame $h_{i}$ dual to $b^{j}$ is defined by $\left.h_{i}\right\lrcorner b^{j}=\delta_{i}^{j}$, the signature of the metric is $(+,-,-)$, totally antisymmetric symbol $\varepsilon^{i j k}$ is normalized to $\varepsilon^{012}=1$, the Lie dual of an antisymmetric form $X^{i j}$ is $X_{i}:=-\varepsilon_{i j k} X^{j k} / 2$, the Hodge dual of a form $\alpha$ is ${ }^{\star} \alpha$, and the exterior product of forms is implicit.

\section{Conformally flat Riemannian solutions in PGT}

The OTT black hole is a vacuum solution of the BHT gravity with a unique AdS ground state [12, 14]. Here, based on our earlier experience [8, 9], we wish to interpret it as a Riemannian solution of PGT in vacuum. By doing so, we will be able to use the full power of the constrained Hamiltonian formalism to clarify the asymptotic structure and find the conserved charges for both the static and the rotating OTT black hole.

The possibility to interpret the OTT black hole as a Riemannian solution of PGT (a solution with vanishing torsion) is not just a coincidence, it is based on a deep dynamical relation between the PGT sector of Riemannian solutions and the BHT gravity. The content of this relation is expressed by a theorem stating that any conformally flat solution of the BHT gravity is also a Riemannian solution of PGT. This is, in particular, true for the OTT black holes. In 3D, the Weyl curvature identically vanishes, and the Cotton 2form $C^{i}$ is used to characterize conformal properties of spacetime [19]. It is defined by 
$C^{i}:=\nabla L^{i}=d L^{i}+\omega_{m}^{i} L^{m}$ where $L^{m}:=R i c^{m}-\frac{1}{4} R b^{m}$ is the Schouten 1-form. A spacetime is conformally flat when $C^{i}=0$.

To prove the above theorem, we note that the BHT gravity action

$$
I_{\mathrm{BHT}}=a_{0} \int d^{3} x \sqrt{g}\left(R-\lambda+\frac{1}{m^{2}} K\right), \quad K:=R i c^{i j} \operatorname{Ric}_{i j}-\frac{3}{8} R^{2},
$$

leads to the field equations [21]:

$$
\begin{aligned}
& G_{i j}-\lambda \eta_{i j}-\frac{1}{2 m^{2}} K_{i j}=0, \\
& K_{i j}=K \eta_{i j}-2 L_{i k} G^{k}{ }_{j}-2\left(\nabla_{m} C_{i n}\right) \varepsilon^{m n},
\end{aligned}
$$

where $G_{i j}=R i c_{i j}-R \eta_{i j} / 2$ is the Einstein tensor, $\left.C_{i j}=h_{j}\right\lrcorner^{\star} C_{i}$ is the Cotton and $L_{i j}=$ $\left.h_{j}\right\lrcorner L_{i}$ the Schouten tensor. This compact form of the BHT field equations significantly simplifies the analysis of conformally flat solutions.

Lagrangian dynamics of PGT is expressed in terms of its basic field variables, the triad $b^{i}$ and the Lorentz connection $\omega^{i j}$ (1-forms), the related field strengths are the torsion $T^{i}:=d b^{i}+\omega_{m}^{i} b^{m}$ and the curvature $R^{i j}:=d \omega^{i j}+\omega^{i}{ }_{m} \omega^{m j}$ (2-forms), and the spacetime continuum is described by a Riemann-Cartan geometry. The gravitational Lagrangian $L_{G}=L_{G}\left(b^{i}, T^{j}, R^{m n}\right)$ (3-form) is at most quadratic in the field strengths:

$$
\begin{aligned}
L_{G}= & -{ }^{\star}\left(a_{0} R+2 \Lambda_{0}\right)+T^{i \star}\left(a_{1}{ }^{(1)} T_{i}+a_{2}{ }^{(2)} T_{i}+a_{3}{ }^{(3)} T_{i}\right) \\
& +\frac{1}{2} R^{i j \star}\left(b_{4}{ }^{(4)} R_{i j}+b_{5}{ }^{(5)} R_{i j}+b_{6}{ }^{(6)} R_{i j}\right),
\end{aligned}
$$

where ${ }^{(n)} T^{i}$ and ${ }^{(n)} R^{i j}$ are irreducible components of the respective field strengths, and $a_{0}$ is normalized by $a_{0}=/ 16 \pi G$; for details, see Ref. [7]. Since we are here interested only in Riemannian solutions of PGT, the torsion can be effectively set to vanish, whereas the curvature becomes Riemannian; in 3D, it has only two nonvanishing irreducible components,

$$
{ }^{(6)} R^{i j}=\frac{1}{6} R b^{i} b^{j}, \quad{ }^{(4)} R_{i j}=R^{i j}-{ }^{(6)} R^{i j},
$$

whereas the third one vanishes, ${ }^{(5)} R_{i j}=0$. The Riemannian reduction of the general field equations takes the form derived in Appendix A of Ref. [9]:

$$
\begin{array}{cl}
(1 \mathrm{ST}) & E_{i}=0, \\
(2 \mathrm{ND}) & \nabla H_{i j}=0,
\end{array}
$$

where

$$
\begin{aligned}
& \left.\left.E_{i}=h_{i}\right\lrcorner L_{G}-\frac{1}{2}\left(h_{i}\right\lrcorner R^{m n}\right) H_{m n}, \\
& H_{i j}=-2 a_{0} \varepsilon_{i j m} b^{m}+\frac{b_{4}+2 b_{6}}{6} R \varepsilon_{i j k} b^{k}-2 b_{4} \varepsilon_{i j}{ }^{m} L_{m} .
\end{aligned}
$$

Let us now note a simple property of (2ND): the vanishing of the second term in $H_{i j}$ implies that the Cotton 2-form $C_{m}=\nabla L_{m}$ vanishes. More precisely: 
(T1) A Riemannian solution of PGT is conformally flat iff $b_{4}+2 b_{6}=0$.

Next, to examine the content of (1ST), it is convenient to express it in the tensorial form:

$$
a_{0} \operatorname{Ric}_{i j}+2 \Lambda_{0} \eta_{i j}+b_{4} L_{i m} G^{m}{ }_{j}=0 .
$$

In combination with its trace, $a_{0} R+6 \Lambda_{0}+b_{4} K=0$, it can be transformed to

$$
a_{0} G_{i j}-\Lambda_{0} \eta_{i j}-b_{4} \frac{1}{2}\left(K \eta_{i j}-2 L_{i m} G_{j}^{m}\right)=0 .
$$

A direct comparison shows that Eq. (2.3) coincides with the BHT field equation (2.1) for $C_{i n}=0$, provided one makes the following identification of parameters:

$$
\Lambda_{0}=a_{0} \lambda, \quad b_{4}=a_{0} / m^{2} .
$$

This leads to the main result of this section:

(T2) Any conformally flat solution of the BHT gravity is also a Riemannian solution of PGT with $b_{4}+2 b_{6}=0$, and vice versa.

An interesting interpretation of the identifications (2.4) is found by using the BHT condition $\lambda=-m^{2}$ that ensures the existence of the unique maximally symmetric background. For $m^{2}=1 / 2 \ell^{2}$, the identifications (2.4) are transformed into

$$
\Lambda_{0}=-a_{0} / 2 \ell^{2}, \quad b_{4}=2 a_{0} \ell^{2} .
$$

Theorems (T1) and (T2) allow us to study conformally flat solutions of the BHT massive gravity relying on the powerful Hamiltonan methods developed in the context of PGT [2, 4, 11]. In particular, we will use these methods to study boundary terms, conserved charges, and central charges of the OTT black hole. Recently, it was shown by Barnich et al. 20] that BHT gravity admits black hole solutions that can be deformed into dynamical "black flowers", a new class of solutions that are no longer spherically symmetric. Since black flowers are conformally flat, they are also solutions of PGT.

Although PGT is used here as a convenient framework for studying conformally flat solutions of the BHT gravity, it is worth mentioning some general dynamical aspects of PGT, expressed through its unitarity properties. In 3D, the requirement of unitary propagation of torsion modes leads to certain conditions on the coupling constants, the form of which is given in Eqs. (17) of Ref. [6]. The content of these equations leads to the following conclusions: (a) the condition $b_{4}+2 b_{6}=0$ implies that the spin- $0^{+}$mode does not propagate; (b) for a suitable choice of the remaining coupling constants, the propagation of the spin- $0^{-}$, spin-1 or spin-2 modes is unitary.

\section{Static OTT black hole}

Now, we turn our attention to the static OTT spacetime, described by the metric [12]

$$
d s^{2}=N^{2} d t^{2}-\frac{d r^{2}}{N^{2}}-r^{2} d \varphi^{2}, \quad N^{2}:=-\mu+b r+\frac{r^{2}}{\ell^{2}}
$$


where $\mu$ and $b$ are real parameters. The roots of equation $N^{2}=0$ are

$$
r_{ \pm}=\frac{1}{2}\left(-b \ell^{2} \pm \ell \sqrt{4 \mu+b^{2} \ell^{2}}\right)
$$

The OTT metric defines a static AdS black hole when $\ell^{2}>0$ and at least $r_{+}$is real and positive; for $b=0$ it reduces to the BTZ black hole [22].

In order to have a suitable geometric description of the OTT black hole in the framework of PGT, we introduce the triad field (1-form)

$$
b^{0}:=N d t, \quad b^{1}:=\frac{d r}{N}, \quad b^{2}:=r d \varphi,
$$

so that $d s^{2}=\eta_{i j} b^{i} \otimes b^{j}$, with $\eta=\operatorname{diag}(+1,-1,-1)$, and the corresponding Riemannian connection (1-form):

$$
\omega^{01}=-N^{\prime} b^{0}, \quad \omega^{02}=0, \quad \omega^{12}=\frac{N}{r} b^{2},
$$

where $N^{\prime}:=\partial_{r} N$. The geometric structure introduced in Eqs. (3.2) can be now used to calculate first the curvature 2-form $R^{i j}$, and then the Schouten 1-form:

$$
L^{0}=\frac{1}{2 \ell^{2}} b^{0}, \quad L^{1}=\frac{1}{2 \ell^{2}} b^{1}, \quad L^{2}=\left(\frac{1}{2 \ell^{2}}+\frac{b}{2 r}\right) b^{2} .
$$

An explicit calculation yields $C^{i}=\nabla L^{i}=0$, and theorem (T2) from section 2 implies that the static OTT black hole is an exact Riemannian solution of PGT in vacuum.

It is interesting to compare these general arguments with direct calculations based on the PGT field equations (2.2). As shown in [9], the result takes the form of three conditions on the four Lagrangian parameters $\left(a_{0}, b_{4}, b_{6}, \Lambda\right)$ :

$$
b_{4}-2 a_{0} \ell^{2}=0, \quad a_{0}+2 \ell^{2} \Lambda_{0}=0, \quad b_{4}+2 b_{6}=0 .
$$

The meaning of these conditions is now quite clear: the third one follows from the conformal flatness of the static OTT black hole, and the first two coincide with the relations (2.5).

\section{Asymptotic structure of the static black hole}

In this section, we use the canonical approach to analyze the asymptotic structure naturally associated to the static OTT black hole. In particular, we wish to calculate the conserved charges and verify their compatibility with the first law of black hole thermodynamics.

\subsection{Asymptotic conditions}

The asymptotic state associated to the triad (

$$
N=\frac{r}{\ell}+\frac{b \ell}{2}-\frac{\ell}{2 r}\left(\mu+\frac{b^{2} \ell^{2}}{4}\right)+\mathcal{O}_{2}
$$


and a similar formula for $1 / N$. In order to produce a suitable set of the asymptotic states, we act on this particular state by the transformations belonging to the AdS group $S O(2,2)$, as described in Ref. [7. The family of triads obtained in this way has the AdS asymptotic behavior given by $b^{i}{ }_{\mu}=\bar{b}^{i}{ }_{\mu}+B^{i}{ }_{\mu}$, where

$$
\bar{b}_{\mu}^{i}:=\left(\begin{array}{ccc}
\frac{r}{\ell} & 0 & 0 \\
0 & \frac{\ell}{r} & 0 \\
0 & 0 & r
\end{array}\right), \quad B_{\mu}^{i}:=\left(\begin{array}{ccc}
\mathcal{O}_{0} & \mathcal{O}_{3} & \mathcal{O}_{0} \\
\mathcal{O}_{1} & \mathcal{O}_{2} & \mathcal{O}_{1} \\
\mathcal{O}_{0} & \mathcal{O}_{3} & \mathcal{O}_{0}
\end{array}\right) .
$$

Here, $\bar{b}_{\mu}^{i}$ refers to an AdS background $(b=\mu=0)$. Note that the presence of the OTT parameter $b$ makes the asymptotic decrease of $B^{i}{ }_{\mu}$ slower then in the BTZ case. The subset of the local Poincaré transformations that respect these conditions is determined by the parameters $\left(\xi^{\mu}, \varepsilon^{i j}=-\varepsilon^{i j k} \theta_{k}\right)$, such that

$$
\delta_{0} b_{\mu}^{i}:=\varepsilon^{i j k} \theta_{j} b_{k \mu}-\left(\partial_{\mu} \xi^{\rho}\right) b_{\rho}^{i}-\xi^{\rho} \partial_{\rho} b_{\mu}^{i}=B_{\mu}^{i} .
$$

As a consequence, the asymptotic parameters of local translations and Lorentz rotations are found to be

$$
\begin{aligned}
\frac{\xi^{t}}{\ell} & =T+\frac{\ell^{4}}{2 r^{2}} \partial_{t}^{2} T+\mathcal{O}_{3}, \quad \xi^{r}=-\ell r \partial_{t} T+\mathcal{O}_{0}, \\
\xi^{\varphi} & =S-\frac{\ell^{2}}{2 r^{2}} \partial_{\varphi}^{2} S+\mathcal{O}_{3}, \\
\theta^{0} & =-\frac{\ell^{2}}{r} \partial_{t} \partial_{\varphi} T+\mathcal{O}_{2}, \quad \theta^{1}=\partial_{\varphi} T+\mathcal{O}_{1}, \\
\theta^{2} & =\frac{\ell^{3}}{r} \partial_{t}^{2} T+\mathcal{O}_{2} .
\end{aligned}
$$

The functions $T$ and $S$ are such that $\partial_{ \pm} T^{\mp}=0$, with $x^{ \pm}:=t / \ell \pm \varphi$, and $T^{ \pm}:=T \pm S$. Thus, in spite of a relaxed asymptotic behavior of $B_{\mu}^{i}$ as compared to the BTZ black hole, the values of the corresponding asymptotic parameters are essentially the same [23].

Similar procedure leads to the asymptotic conditions for the connection. Introducing the Lie dual connection $\omega^{i}$ by $\omega^{i j}=-\varepsilon^{i j k} \omega_{k}$, one finds $\omega^{i}{ }_{\mu}=\bar{\omega}^{i}{ }_{\mu}+\Omega^{i}{ }_{\mu}$, where

$$
\bar{\omega}_{\mu}^{i}=\left(\begin{array}{lll}
0 & 0 & -\frac{r}{\ell} \\
0 & 0 & 0 \\
-\frac{r}{\ell^{2}} & 0 & 0
\end{array}\right), \quad \Omega_{\mu}^{i}:=\left(\begin{array}{ccc}
\mathcal{O}_{0} & \mathcal{O}_{3} & \mathcal{O}_{0} \\
\mathcal{O}_{1} & \mathcal{O}_{2} & \mathcal{O}_{1} \\
\mathcal{O}_{0} & \mathcal{O}_{3} & \mathcal{O}_{0}
\end{array}\right) .
$$

The asymptotic behavior of the connection does not impose any new restriction on the asymptotic Poincaré parameters (4.2).

For an easier comparison with the literature, we display here the deviation of the metric from its background value:

$$
G_{\mu \nu}:=g_{\mu \nu}-\bar{g}_{\mu \nu}=\left(\begin{array}{lll}
\mathcal{O}_{-1} & \mathcal{O}_{2} & \mathcal{O}_{-1} \\
\mathcal{O}_{2} & \mathcal{O}_{3} & \mathcal{O}_{2} \\
\mathcal{O}_{-1} & \mathcal{O}_{2} & \mathcal{O}_{-1}
\end{array}\right)
$$


Using the composition law of the asymptotic Poincaré parameters (4.2) to leading order, the commutator algebra of the asymptotic symmetry is found to have the form of two independent Virasoro algebras,

$$
i\left[\ell_{m}^{ \pm}, \ell_{n}^{ \pm}\right]=(m-n) \ell_{m+n}
$$

where $\ell_{n}^{ \pm}=-\delta_{0}\left(T^{ \pm}=e^{ \pm i n x^{ \pm}}\right)$. The respective central charges $c^{ \pm}$will be determined by the canonical methods.

The condition $T^{i}=0$ leads to further asymptotic requirements (Appendix A).

\subsection{Canonical generator and conserved charges}

The standard construction of the canonical generator for the quadratic PGT makes use of the existence and classification of all constraints in the theory. The construction can be significantly simplified by going over to the first order Lagrangian (3-form)

$$
L_{G}=T^{i} \tau_{i}+\frac{1}{2} R^{i j} \rho_{i j}-V(b, \tau, \rho),
$$

see Refs. [11, 25]. Here, $\tau^{m}$ and $\rho_{i j}$ are independent dynamical variables, the covariant field momenta conjugate to $b^{i}$ and $\omega^{i j}$, and the potential $V$ ensures the on-shell relations $\tau_{i}=T_{i}$, $\rho_{i j}=R_{i j}$, which transform $L_{G}$ into the standard quadratic form.

The first order formulation of $L_{G}$ simplifies the construction of the canonical generator $G$, the form of which can be found in Ref. [7], equation (5.7). Since $G$ acts on the basic dynamical variables via the Poisson bracket operation, it must be a differentiable functional. To examine the differentiability of $G$, one starts from the form of its variation [8, 9]:

$$
\begin{aligned}
& \delta G=-\int_{\Sigma} d^{2} x\left(\delta G_{1}+\delta G_{2}\right) \\
& \delta G_{1}=\varepsilon^{t \alpha \beta} \xi^{\mu}\left(b^{i}{ }_{\mu} \partial_{\alpha} \delta \tau_{i \beta}+\omega^{i}{ }_{\mu} \partial_{\alpha} \delta \rho_{i \beta}+\tau_{\mu}^{i} \partial_{\alpha} \delta b_{i \beta}+\rho_{\mu}^{i} \partial_{\alpha} \delta \omega_{i \beta}\right)+\mathcal{R} \\
& \delta G_{2}=\varepsilon^{t \alpha \beta} \theta^{i} \partial_{\alpha} \delta \rho_{i \beta}+\mathcal{R}
\end{aligned}
$$

Here, $\Sigma$ is the spatial section of spacetime, the variation is performed in the set of adopted asymptotic states, $\mathcal{R}$ stands for regular (differentiable) terms, and we use $\rho^{i}$ and $\omega^{i}$, the Lie duals of $\rho_{m n}=H_{m n}$ and $\omega_{m n}$, to simplify the formulas.

Using the adopted asymptotic conditions, one finds $\delta G_{2}=\mathcal{R}$, which implies

$$
\delta G=-\int_{\Sigma} d^{2} x \varepsilon^{t \alpha \beta} \xi^{\mu}\left(b_{\mu}^{i} \partial_{\alpha} \delta \tau_{i \beta}+\omega_{\mu}^{i} \partial_{\alpha} \delta \rho_{i \beta}+\tau_{\mu}^{i} \partial_{\alpha} \delta b_{i \beta}+\rho_{\mu}^{i} \partial_{\alpha} \delta \omega_{i \beta}\right)+\mathcal{R} .
$$

Thus, in general, $\delta G \neq \mathcal{R}$ and $G$ is not differentiable. The problem can be corrected by going over to the improved generator $\tilde{G}:=G+\Gamma$, where the boundary term $\Gamma$ is constructed so that $\delta \tilde{G}=\mathcal{R}[17]$. After making a partial integration in $\delta G$, one finds that $\Gamma$ is defined by the variational equation

$$
\delta \Gamma=\int_{\partial \Sigma} \xi^{\mu}\left(b_{\mu}^{i} \delta \tau_{i}+\omega_{\mu}^{i} \delta \rho_{i}+\tau_{\mu}^{i} \delta b_{i}+\rho_{\mu}^{i} \delta \omega_{i}\right),
$$


where $\partial \Sigma$ is the boundary of $\Sigma$ located at infinity, parametrized by the coordinate $\varphi$. Now, restricting our attention to the Riemannian sector with $\tau^{i}=0$, we obtain

$$
\delta \Gamma=\int_{\partial \Sigma} \xi^{\mu}\left(\omega^{i}{ }_{\mu} \delta \rho_{i}+\rho_{\mu}^{i} \delta \omega_{i}\right)=\int_{0}^{2 \pi}\left(\xi^{t} \delta \mathcal{E}+\xi^{\varphi} \delta \mathcal{J}\right) d \varphi,
$$

where (after returning to $\omega_{i j}$ and $H_{i j}$ )

$$
\begin{aligned}
\delta \mathcal{E} & :=\frac{1}{2}\left(\omega^{i j}{ }_{t} \delta H_{i j \varphi}+H^{i j}{ }_{t} \delta \omega_{i j \varphi}\right) \\
\delta \mathcal{J} & :=\frac{1}{2}\left(\omega^{i j}{ }_{\varphi} \delta H_{i j \varphi}+H^{i j}{ }_{\varphi} \delta \omega_{i j \varphi}\right) .
\end{aligned}
$$

In what follows, one should take into account that the form (2.2b) of $H_{m n}$ is simplified after using the restrictions (3.4) on the Lagrangian parameters:

$$
H_{i j}=-2 a_{0} \varepsilon_{i j k} b^{k}-4 a_{0} \ell^{2} \varepsilon_{i j k} L^{k} .
$$

Once we find the solutions for $\mathcal{E}$ and $\mathcal{J}$, the boundary term takes the form

$$
\Gamma(\xi)=\int_{0}^{2 \pi}\left(\xi^{t} \mathcal{E}+\xi^{\varphi} \mathcal{J}\right) d \varphi
$$

In general, Eqs. (4.7) refer to the fields and their variations belonging to the entire set of asymptotic states, defined by Eqs. (4.1) and (4.3). However, it is instructive to consider first a simpler situation, in which the fields and their variations refer just to a single asymptotic state, the static OTT configuration (3.2). In that case, Eq. (4.7b) takes the form

$$
\begin{aligned}
\delta \mathcal{E} & =\omega^{01}{ }_{t} \delta H_{01 \varphi}+H^{12}{ }_{t} \delta \omega_{12 \varphi} \\
& =2 a_{0} \ell^{2}\left(\frac{r}{\ell^{2}}+\frac{1}{2} b\right) \delta b-4 a_{0} N \delta N \\
& =2 a_{0} \delta\left(\mu+\frac{1}{4} \ell^{2} b^{2}\right),
\end{aligned}
$$

that is easily integrated to obtain $\mathcal{E}$. In fact, the procedure just described is sufficient to calculate the values of the conserved charges, but only for this particular configuration.

In the next step, we wish to find a solution for $\mathcal{E}$ on the whole set of asymptotic states. Using the special result (4.9) as a guide, we find

$$
\begin{aligned}
& \mathcal{E}=\mathcal{E}_{0}-\frac{1}{4}\left(\Delta \omega^{i j}{ }_{t} \Delta H_{i j \varphi}+\Delta H_{i j t} \Delta \omega^{i j}{ }_{\varphi}\right), \\
& \mathcal{E}_{0}:=\frac{1}{2}\left(\omega^{i j}{ }_{t} \Delta H_{i j \varphi}+H_{i j t} \Delta \omega^{i j}{ }_{\varphi}\right),
\end{aligned}
$$

where $\Delta X:=X-\bar{X}$ is the difference between any field $X$ and its boundary value $\bar{X}$. In a similar manner, Eq. (4.7c) leads to

$$
\begin{aligned}
\mathcal{J} & =\frac{1}{2} \omega^{i j}{ }_{\varphi} H_{i j \varphi}=\mathcal{J}_{0}-\frac{1}{2} \Delta H_{i j \varphi} \Delta \omega^{i j}{ }_{\varphi}, \\
\mathcal{J}_{0} & :=\frac{1}{2}\left(\omega^{i j}{ }_{\varphi} \Delta H_{i j \varphi}+H_{i j \varphi} \Delta \omega^{i j}{ }_{\varphi}\right),
\end{aligned}
$$


where the first equality follows directly from (4.7c), and the second one from $\bar{H}_{i j \varphi} \bar{\omega}^{i j}{ }_{\varphi}=0$. With these results for $\mathcal{E}$ and $\mathcal{J}$, the boundary term (4.8) is seen to to be a finite phase-space functional that satisfies the variational equation (4.7a) (Appendix B).

The values of the improved generators for time translations $\left(\xi=\partial_{t}\right)$ and spatial rotations $\left(\xi=\partial_{\varphi}\right)$ are given by the corresponding boundary terms, which define the conserved charges of the system, the energy and the angular momentum, respectively:

$$
E=\int_{0}^{2 \pi} d \varphi \mathcal{E}, \quad J=\int_{0}^{2 \pi} d \varphi \mathcal{J},
$$

Calculated on the static OTT configuration, these expressions take the values

$$
E=\frac{1}{4 G}\left(\mu+\frac{1}{4} b^{2} \ell^{2}\right), \quad J=0 .
$$

The expressions (4.10) for $\mathcal{E}$ and $\mathcal{J}$ are obtained by relying on the set of asymptotic configurations (4.1) and (4.3) that contain the static OTT black hole geometry. It is interesting to compare the boundary term (4.8) to the covariant approach of Chen et al. [11]. Looking at the Riemannian reduction of their formula (239) and choosing the upper or lower term in each curly bracket separately, one finds that none of the resulting expressions can reproduce our result. To make the argument more clear, consider, for instance, the term $\mathcal{E}_{0}$ in (4.10a) that corresponds to choosing all the upper terms in (239); the corresponding expression for the energy would be different from (4.12): $E_{0}=\frac{1}{4 G}\left(\mu+\frac{1}{2} b^{2} \ell^{2}\right)$. How do we know that this result is not correct? The answer can be found by noting that the boundary term $\Gamma[\xi]$ has a twofold role: (i) its values define the conserved charges, and (ii) its form ensures the improved generator $\tilde{G}=G+\Gamma$ to be a differentiable functional on the phase space associated with the chosen boundary conditions. Since $\mathcal{E}_{0}$ does not satisfy the variational equation $(4.7 \mathrm{~b})$, replacing $\mathcal{E}$ by $\mathcal{E}_{0}$ would destroy the differentiability of the new canonical generator $\tilde{G}\left[\mathcal{E} \rightarrow \mathcal{E}_{0}\right]$. The way out of this situation can be found in the work of So [18], who proposed a generalized boundary term by introducing "mixed" choices involving a linear combinations of upper and lower term in (239), see footnote "u" in [11]. As discussed in section 6, our boundary term (4.8) is appropriately described by a particular mixed form. The need for using a mixed boundary term stems directly from the slower asymptotic decrease of the OTT dynamical variables as compared to the BTZ case (see subsection 4.1), or equivalently, from the presence of the $b r$ term in the OTT metric (3.1).

\subsection{Asymptotic symmetry}

The results obtained so far allow us to precisely describe the OTT asymptotic symmetry by the Poisson bracket algebra of the improved canonical generators. Following the procedure described in [8, 9], one finds that this algebra, expressed in terms of the Fourier modes $L_{n}^{ \pm}$ of $\tilde{G}$, is given by a centrally extended form of the commutator algebra (4.4),

$$
i\left[L_{m}^{ \pm}, L_{n}^{ \pm}\right]=(m-n) L_{m+n}+\frac{c^{ \pm}}{12} m^{3} \delta_{m,-n},
$$

where $c^{ \pm}$are classical central charges,

$$
c^{ \pm}=c, \quad c=\frac{3 \ell}{G} .
$$




\subsection{Black hole entropy}

As an additional, theoretical test of the validity of our canonical expression for the OTT energy (4.12) 1 , we propose to verify its exact agreement with the first law of black hole thermodynamics; the same strategy was used, for instance, by Giribet and Leston [15], and by Maeda [26].

The black hole entropy can be calculated from the Cardy formula [24]

$$
S=2 \pi \sqrt{\frac{h^{-} c^{-}}{6}}+2 \pi \sqrt{\frac{h^{+} c^{+}}{6}},
$$

where $h^{ \pm}=(\ell E \pm J) / 2$. For the static OTT black hole, this formula yields

$$
S=2 \pi \ell \sqrt{\frac{E}{G}} .
$$

Then, using the expression for the Hawking temperature,

$$
T=\left.\frac{1}{4 \pi} \partial_{r} N^{2}\right|_{r=r_{+}}=\frac{1}{\pi \ell} \sqrt{G E},
$$

one can directly verify the first law of the black hole thermodynamics:

$$
\delta E=T \delta S .
$$

Since the entropy vanishes for $E=0$, the state with $E=0$ can be naturally regarded as the ground state of the OTT family of black holes [14.

\section{$5 \quad$ Rotating OTT black hole}

In order to verify to what extent the canonical expressions (4.10) for the boundary terms of the static OTT black hole are general, we now use the same approach to study the rotating OTT black hole.

\subsection{Geometric aspects}

The rotating OTT black hole is defined by the metric [14, 15]

$$
d s^{2}=N^{2} d t^{2}-F^{-2} d r^{2}-r^{2}\left(d \varphi+N_{\varphi} d t\right)^{2}
$$

where

$$
\begin{aligned}
& F=\frac{H}{r} \sqrt{\frac{H^{2}}{\ell^{2}}+\frac{b}{2} H(1+\eta)+\frac{b^{2} \ell^{2}}{16}(1-\eta)^{2}-\mu \eta} \\
& N=A F, \quad A=1+\frac{b \ell^{2}}{4 H}(1-\eta), \\
& N_{\varphi}=\frac{\ell}{2 r^{2}} \sqrt{1-\eta^{2}}(\mu-b H) \\
& H=\sqrt{r^{2}-\frac{\mu \ell^{2}}{2}(1-\eta)-\frac{b^{2} \ell^{4}}{16}(1-\eta)^{2}} .
\end{aligned}
$$


The roots of $N=0$ are

$$
r_{ \pm}=\ell \sqrt{\frac{1+\eta}{2}}\left(-\frac{b \ell}{2} \sqrt{\eta} \pm \sqrt{\mu+\frac{b^{2} \ell^{2}}{4}}\right) .
$$

The metric (5.1) depends on three free parameters, $\mu, b$ and $\eta$. For $\eta=1$, it represents the static OTT black hole, and for $b=0$, it reduces to the rotating BTZ black hole with parameters $(m, j)$, such that $4 G m:=\mu$ and $4 G j:=\mu \ell \sqrt{1-\eta^{2}}$.

Choosing the triad field as

$$
b^{0}=N d t, \quad b^{1}=F^{-1} d r, \quad b^{2}=r\left(d \varphi+N_{\varphi} d t\right),
$$

the Riemannian connection takes the form

$$
\omega^{01}=-\alpha b^{0}+\beta b^{2}, \quad \omega^{02}=\beta b^{1}, \quad \omega^{12}=-\beta b^{0}+\gamma b^{2},
$$

where $\alpha:=F N^{\prime} / N, \beta:=r F N_{\varphi}^{\prime} / 2 N$ and $\gamma=F / r$. These objects define the Riemannian geometry of the rotating OTT black hole in the context of PGT.

Now, based on theorem (T2) from section 2, we know that the rotating OTT black hole, being an exact solution of the BHT gravity, is also a solution of PGT provided its Cotton tensor vanishes. Technically, the proof that $C^{i j}=0$ is not quite simple due to the complicated structure of the metric functions $N, F$ and $N_{\varphi}$. However, relying on the standard computer algebra systems, one easily finds that $C^{i j}$ indeed vanishes.

\subsection{Asymptotic conditions and conserved charges}

A direct inspection of the rotating black hole geometry (5.2) shows that it belongs to the same class of asymptotic states as described by Eqs. (4.1) and (4.3). Hence, the results for (i) the boundary term (4.8), and (ii) the classical central charges (4.14), remain valid also in the rotating black hole case.

Applying formulas (4.10) to the rotating OTT geometry (5.2) yields the following conserved charges:

$$
\begin{aligned}
& E=\frac{1}{4 G}\left(\mu+\frac{1}{4} b^{2} \ell^{2}\right), \\
& J=\ell \sqrt{1-\eta^{2}} E .
\end{aligned}
$$

For $\eta=1$ the angular momentum vanishes, whereas for $b=0$ we have the BTZ black hole with $E=m$ and $J=j$; its energy is twice as big as in GR.

\subsection{The first law of black hole thermodynamics}

The entropy for the rotating OTT black hole can be calculated in the same manner as for the static one. Using the above expressions for $E, J$, and the central charges $c^{ \pm}=3 \ell / G$, the Cardy formula yields

$$
S=2 \pi \ell \sqrt{\frac{(1+\eta) E}{2 G}} .
$$


The Hawking temperature and the angular velocity at the outer horizon are:

$$
\begin{aligned}
& T=\left.\frac{1}{4 \pi} \frac{\partial_{r} N^{2}}{A}\right|_{r=r_{+}}=\frac{1}{\pi \ell} \sqrt{\frac{2 \eta^{2}}{1+\eta}} \sqrt{G E}, \\
& \Omega_{+}=\left.N_{\varphi}\right|_{r=r_{+}}=\frac{1}{\ell} \sqrt{\frac{1-\eta}{1+\eta}} .
\end{aligned}
$$

Then, the first law of black hole thermodynamics is automatically satisfied:

$$
T \delta S=\delta E-\Omega_{+} \delta J
$$

\section{Discussion and conclusions}

The OTT black hole energy was calculated already in the original paper [12], based on the Deser-Tekin approach [27]. Since the Deser-Tekin formula (37) in [12] does not contain the asymptotic terms produced by the parameter $b$, the resulting energy $E_{\mathrm{DT}}=\mu / 4 G$ does not depend on $b$. This result is evidently not compatible with the first law of black hole thermodynamics. Then, Giribet et al. [14] found certain arguments, based on interpreting $b$ as a 'hair' parameter, to transform $E_{\mathrm{DT}}$ into $E=\left(\mu+\ell^{2} b^{2} / 4\right) /(4 G)$, the expression that is fully compatible with the first law [26].

In the next paper, Giribet and Leston [15] tried to find more convincing arguments to derive the above form of $E$. Their approach was based on the work of Hohm and Tonni [28], who developed a generalized Brown-York approach to the generic form of the BHT gravity. By restricting their considerations to the special value of $\mathrm{m}^{2}$, where the OTT black hole is admitted as an exact solution, the authors of [15] succeeded to derive the above result for $E$, but only for the rotating black hole, where certain ambiguity in the derivation disappears. By improving the construction, Kwon et al. [16] obtained the conserved charges for both the static and the rotating OTT black hole. Our expressions (5.3) for the conserved charges confirm their final results, given in Eq. (44).

In the approach initiated by Regge and Teitelboim [17], the gravitational conserved charges and the improved canonical generators are closely related to each other. An important progress in understanding essential aspects of this relation has been achieved in the first order approach, which allows one to find a covariant boundary term and identify its value as a conserved charge; for an early version of the formalism, see Nester [25], and for a comprehensive exposition of this approach, see Chen et al. [11. The covariant approach has been widely used in 4D gauge theories of gravity with a great success [4, 11]. Moreover, it was also confirmed on a set of selected 3D solutions [29]. Now, in order to properly understand our results in the context of this approach, we start from a particular choice of the covariant boundary expression (integrand) defined by the upper line in Eq. (234) of [11]:

$$
\left.\left.\left.\left.B_{\mathrm{ul}}(\xi):=(\xi\lrcorner b^{i}\right) \Delta \tau_{i}+\Delta b^{i}(\xi\lrcorner \tau_{i}\right)+(\xi\lrcorner \omega^{i}\right) \Delta \rho_{i}+\Delta \omega^{i}(\xi\lrcorner \rho_{i}\right) .
$$

Here, $\Delta X=X-\bar{X}$ is a difference between a field $X$ and its boundary value $\bar{X}$, and $\xi$ is asymptotically a Killing vector field. The lower line is obtained by replacing the variables $\left(b^{i}, \tau_{i}, \omega^{i}, \rho_{i}\right)$ with their boundary values. One can verify that formula (6.1), taken in the 
Riemannian limit, is not compatible with our result (4.8). This is in fact true for all sixteen versions of $B(\xi)$, obtained from Eq. (234) of [11] by choosing either upper or lower term in each of the four curly brackets separately. However, the situation is changed by generalizing the construction of $B(\xi)$ in a way proposed by So [18. According to his prescription, the original Hamiltonian boundary term $B(\xi)$ is modified by replacing each curly bracket by a linear combination of its upper and lower term. Applying this prescription to Eq. (234) of [11], one finds that its Riemannian reduction takes the form

$$
\left.\left.\tilde{B}\left(\xi ; c_{3}, c_{4}\right):=\xi\right\lrcorner\left[c_{3} \omega^{i}+\left(1-c_{3}\right) \bar{\omega}^{i}\right] \wedge \Delta \rho_{i}+\Delta \omega^{i} \wedge \xi\right\lrcorner\left[c_{4} \rho_{i}+\left(1-c_{4}\right) \bar{\rho}_{i}\right],
$$

where $c_{3}$ and $c_{4}$ are real parameters. For the particular choice $\left(c_{3}, c_{4}\right)=(1 / 2,1 / 2)$, we have

$$
\left.\tilde{B}(\xi ; 1 / 2,1 / 2):=\xi\lrcorner\left[\omega^{i}-\frac{1}{2} \Delta \omega^{i}\right] \wedge \Delta \rho_{i}+\Delta \omega^{i} \wedge \xi\right\lrcorner\left[\rho_{i}-\frac{1}{2} \Delta \rho_{i}\right],
$$

A comparison with Eqs. (4.10) shows that the boundary term $\int_{\partial \Sigma} \tilde{B}(\xi ; 1 / 2,1 / 2)$ exactly coincides with our expression $\Gamma(\xi)$, Eq. (4.8).

Clearly, the result (6.3) represents only a Riemannian reduction of a more general So-like formula for the boundary term. With an obvious extension of notation, this more general formula can be represented in the form

$$
B(\xi)=B\left(\xi ; c_{1}, c_{2}, 1 / 2,1 / 2\right)
$$

Additional information on the general structure of $B$ can be found in Ref. [28, where the conserved charges of several 3D solutions were calculated. However, the results are not sufficiently sensitive to clearly recognize the general structure of a "good" expression for the boundary term in PGT, in 3D. Further work in this direction is needed.

In conclusion, we summarize our results as follows.

(a) First, we found general criteria that allow us to study conformally flat Riemannian spacetime configurations as solutions of PGT. These criteria are used to show that the OTT black hole, a solution of the BHT gravity, is a Riemannian solution of PGT.

(b) Then, we constructed a natural set of the asymptotic conditions and calculated the conserved charges of the OTT black hole as the values of the Hamiltonian boundary term. The expressions for the conserved charges coincide with those found by of Kwon et al. [25] in the generalized Brown-York approach.

(c) Finally, the obtained results are verified by showing that: (i) the conserved charges are exactly compatible with the first law of black hole thermodynamics, and (ii) our boundary term is in agreement with the generalized covariant formula proposed by So [18]. On the other hand, the OTT black hole appears to be an interesting physical example for the generalized covariant formula.

\section{Acknowledgements}

One of us (M.B.) would like to thank James Nester for a very instructive discussion on the form of covariant boundary terms. This work was supported by the Serbian Science Foundation under Grant No. 171031. The results are checked using the computer algebra systems Reduce and Mathematica. 


\section{A Useful asymptotic relations}

In the Riemannian sector of PGT, the condition $T^{i}=0$, calculated on the asymptotic configurations (4.1) and (4.3), leads to an additional set of asymptotic requirements:

$$
\begin{aligned}
& \frac{r^{2}}{\ell^{2}} B^{1}{ }_{r}-\ell \Omega^{2}{ }_{t}=\mathcal{O}_{1}, \quad B^{1}{ }_{r}-\frac{\ell^{2}}{r^{2}} \Omega^{0}{ }_{\varphi}=\mathcal{O}_{1}, \\
& \frac{r^{2}}{\ell} \Omega^{1}{ }_{r}+\Omega^{2}{ }_{\varphi}=\mathcal{O}_{1}, \quad \frac{r^{2}}{\ell^{2}} \Omega^{1}{ }_{r}+\Omega^{0}{ }_{t}=\mathcal{O}_{1}, \\
& \frac{B^{0}{ }_{\varphi}}{\ell}+\Omega^{2}{ }_{\varphi}+B^{2}{ }_{t}+\ell \Omega^{0}{ }_{t}=\mathcal{O}_{1} .
\end{aligned}
$$

Then, relying on the asymptotic form of the Schouten tensor $L_{i j}$,

$$
\begin{aligned}
& L_{00}=\frac{1}{2 \ell^{2}}-\frac{1}{r \ell}\left(B^{0}{ }_{t}+\frac{r^{2}}{\ell^{2}} B^{1}{ }_{r}\right)+\mathcal{O}_{2}, \\
& L_{11}=-\frac{1}{2 \ell^{2}}+\mathcal{O}_{2}, \\
& L_{22}=-\frac{1}{2 \ell^{2}}+\frac{1}{r \ell}\left(\frac{r^{2}}{\ell^{2}} B^{1}{ }_{r}+\frac{1}{\ell} B^{2}{ }_{\varphi}\right)+\mathcal{O}_{2}, \\
& L_{02}=-\frac{1}{\ell^{2} r} B^{0}{ }_{\varphi}+\frac{r}{\ell^{2}} \Omega^{1}{ }_{r}+\mathcal{O}_{2},
\end{aligned}
$$

one obtains the asymptotic relations

$$
\begin{aligned}
& \Delta H_{i j t}=-4 a_{0} \Omega_{i j \varphi}+\mathcal{O}\left(\frac{\Omega_{i j \varphi}}{r}\right) \\
& \Delta H_{i j \varphi}=-4 a_{0} \ell^{2} \Omega_{i j t}+\mathcal{O}\left(\frac{\Omega_{i j t}}{r}\right) .
\end{aligned}
$$

\section{B Consistency of the boundary term}

In this appendix, we prove the consistency of the Hamiltonian boundary term (4.8) by showing that it is a finite expression that satisfies the variational equations (4.7a). Using the expressions (4.10) for $\mathcal{E}$ and $\mathcal{J}$, as well as the results of Appendix A, we have:

$$
\begin{aligned}
\mathcal{E} & =4 a_{0} \frac{r}{\ell}\left(\Omega^{0}{ }_{\varphi}-\frac{r^{2}}{\ell^{2}} B^{1}{ }_{r}\right)+\mathcal{O}_{0}=\mathcal{O}_{0}, \\
\mathcal{J} & =2 a_{0} \omega^{i}{ }_{\varphi} b_{i \varphi}+4 a_{0} \ell^{2} L_{i j} \omega^{i}{ }_{\varphi} b^{j}{ }_{\varphi} \\
& =-4 a_{0} r\left(\frac{B^{0}{ }_{\varphi}}{\ell}+\Omega^{2}{ }_{\varphi}\right)-4 a_{0} \ell r^{2} L_{02}+\mathcal{O}_{0} \\
& =-4 a_{0} r\left(\Omega^{2}{ }_{\varphi}+\frac{r^{2}}{\ell} \Omega^{1}{ }_{r}\right)+\mathcal{O}_{0}=\mathcal{O}_{0},
\end{aligned}
$$

which completes the proof of finiteness. 
In a similar manner,

$$
\begin{aligned}
\delta \mathcal{E}= & \frac{1}{2}\left(\omega^{i j}{ }_{t} \delta H_{i j \varphi}+\delta \omega_{i j \varphi} H^{i j}{ }_{t}\right) \\
& +\frac{1}{4}\left(\Delta H_{i j \varphi} \delta \omega^{i j}{ }_{t}-\Delta \omega^{i j}{ }_{t} \delta H_{i j \varphi}-\Delta H_{i j t} \delta \omega^{i j}{ }_{\varphi}+\Delta \omega^{i j}{ }_{\varphi} \delta H_{i j t}\right) \\
& =\frac{1}{2}\left(\omega^{i j}{ }_{t} \delta H_{i j \varphi}+\delta \omega_{i j \varphi} H^{i j}{ }_{t}\right)+\mathcal{O}_{1}
\end{aligned}
$$

whereas the proof for $\delta \mathcal{J}$ is trivial. Thus, the variational equation (4.7a) is satisfied.

\section{References}

[1] T. W. B. Kibble, Lorentz invariance and the gravitational field, J. Math. Phys. 2 (1961) 212-221;

D. W. Sciama, On the analogy between charge and spin in general relativity, in: Recent Developments in General Relativity, Festschrift for Infeld (Pergamon Press, Oxford; PWN, Warsaw, 1962) pp. 415-439.

[2] M. Blagojević, Gravitation and Gauge Symmetries (Institute of Physics, Bristol, 2002).

[3] Yu. N. Obukhov, Poincaré gauge gravity: Selected topics, Int. J. Geom. Meth. Mod. Phys. 3 (2006) 95-138.

[4] M. Blagojević and F. W. Hehl (eds.), Gauge Theories of Gravitation, A Reader with Commentaries (Imperial College Press, London, 2013).

[5] E. W. Mielke and P. Baekler, Topological gauge model of gravity with torsion, Phys. Lett. A 156 (1991) 399-403.

[6] J. A. Helayël-Neto, C. A. Hernaski, B. Pereira-Dias, A. A. Vargas-Paredes, and V. J. Vasquez-Otoya, Chern-Simons gravity with (curvature) ${ }^{2}$ - and (torsion) ${ }^{2}$-terms and a basis of degree-of-freedom projection operators, Phys. Rev. D 82 (2010) 064014.

[7] M. Blagojević and B. Cvetković, 3D gravity with propagating torsion: the AdS sector, Phys. Rev. D 85 (2012) 104003 [10 pages].

[8] M. Blagojević and B. Cvetković, Siklos waves with torsion in 3D, JHEP 11 (2014) 141 [16 pages].

[9] M. Blagojević and B. Cvetković, Vaidya-like exact solutions with torsion, JHEP 05 (2015) 101 [15 pages].

[10] M. Blagojević and B. Cvetković, Siklos waves in Poincaré gauge theory, Phys. Rev. D 92 (2015) 024047 [9 pages].

[11] C.-M. Chen, J. M. Nester and R.-S. Tung, Gravitational energy for GR and Poincaré gauge theories: A covariant Hamiltonian approach, Int. J. Mod. Phys. D 24 (2015) 1530026 [73 pages]. 
[12] J. Oliva, D. Tempo, and R. Troncoso, Three-dimensional black holes, gravitational solitons, kinks and wormholes for BHT massive gravity, JHEP 07 (2009) 011 [25 pages].

[13] E. A. Bergshoeff, O. Hohm and P. K. Townsend, Massive gravity in three dimensions, Phys. Rev. Lett. 102 (2009) 201301 [4 pages].

[14] G. Giribet, J. Oliva, D. Tempo, and R. Troncoso, Microscopic entropy of the threedimensional rotating black hole of BHT massive gravity Phys. Rev. D80 (2009) 124046.

[15] G. Giribet and M. Leston, Boundary stress tensor and counterterms for weakened $\mathrm{AdS}_{3}$ asymptotic in New Massive Gravity, JHEP 09 (2010) 070.

[16] Y. Kwon, S. Nam, J-.D. Park, and S-.H. Yi, Holographic renormalization and stress tensors in New massive gravity, JHEP 11 (2011) 029.

[17] T. Regge and C. Teitelboim, Role of surface integrals in the Hamiltonian formulation of general relativity, Ann. Phys. (N.Y) 88 (1974) 286-318.

[18] L. L. So, A modification of the Chen-Nester quasi-local expressions, Int. J. Mod. Phys. D 16 (2007) 875-884.

[19] A. García, F. W. Hehl, Ch. Heinicke, and A. Macías, The Cotton tensor in Riemannian space-times, Class. Quant. Grav. 21 (2004) 1099-1118.

[20] G. Barnich, C. Troessaert, D. Tempo, and R. Troncoso, Asymptotically locally flat spacetimes and dynamical black flowers in three dimensions, arXiv:1512.05410 [hepth].

[21] M. Blagojević and B. Cvetković, Extra gauge symmetries in BHT gravity, JHEP 03 (2011) 139 [13 pages].

[22] M. Banados, C. Teitelboim and J. Zanelli, Black hole in three-dimensional spacetime, Phys. Rev. Lett. 61 (1992) 1849-1851.

[23] M. Blagojevć and B. Cvetković, Canonical structure of 3D gravity with torsion, in: Progress in General Relativity and Quantum Cosmology, vol. 2, ed. Frank Columbus (Nova Science Publishers, New York, 2005) p. 85 [e-print gr-qc/0412134].

[24] J. A. Cardy, Operator content of two-dimensional conformally invariant theories, Nucl. Phys. B 270 (1986) 186-204.

[25] J. M. Nester, A covariant Hamiltonian for gravity theories, Mod. Phys. Lett. A 6 (1991) 2655-2661.

[26] H. Maeda, Black-hole dynamics in BHT massive gravity, JHEP 1102 (2011) 039 [7 pages].

[27] S. Deser and B. Tekin, Energy in generic higher curvature gravity theories, Phys. Rev. D 67 (2003) 084009. 
[28] O. Hohm and E. Tonni, A boundary stress tensor for higher-derivative gravity in AdS and Lifshitz backgrounds, JHEP 04 (2010) 093.

[29] M. Blagojević and B. Cvetković, Conserved charges in 3D gravity, Phys. Rev. D81 (2010) 124024 [7 pages]. 